\title{
Respuesta supraóptima a los inhibidores de PCSK-9: ¿es obligatorio darlos cada 2 semanas? A propósito de un caso
}

\author{
Over optimum response to PCSK-9 inhibitors: ¿is it mandatory to supply \\ them every 2 weeks?, about a case
}

Parra $G A^{1}$, Lindarte $H H^{2}$, Rubio $A F^{2}$

${ }^{1}$ Especialista en Medicina Interna, Endocrinología; profesor asociado
Universidad Autónoma de Bucaramanga (UNAB), FOSCAL.
${ }^{2}$ Estudiante de Medicina, Universidad Autónoma de Bucaramanga
(UNAB).
Fecha de recepción: $16 / 10 / 2019$
Fecha de aceptación: $26 / 11 / 2019$

Resumen

Los inhibidores del PCSK-9 se presentan como una excelente alternativa de tratamiento en aquellos casos de hipercolesterolemia que no alcanzan las metas con tratamiento usual con estatinas de alta potencia y ezetimiba, adicionalmente en los pacientes con intolerancia a estatinas que necesiten tener metas de colesterol LDL. En este caso exponemos la posibilidad de titular el tiempo de aplicación de PCSK-9i de acuerdo con las metas de los niveles de LDL.

Palabras clave: hipercolesterolemia, inhibidores de hidroximetilglutaril-CoA reductasas, evolocumab.

\begin{abstract}
The PCSK-9 inhibitors are presented as an excellent alternative treatment in those cases of hypercholesterolemia that do not reach goals with high intensity statin treatment and ezetimibe, additionally in patients with statin intolerance who need to have LDL cholesterol goals. In this case we expose the possibility to treat to target LDL goals and use PCSK-9i later than 2 weeks.
\end{abstract}

Keywords: hypercholesterolemia, HydroxymethylglutarylCoA Reductase Inhibitors, evolocumab.

\section{Introducción}

El manejo de las dislipidemias constituye una parte esencial del abordaje del riesgo cardiovascular, al ser uno de los más importantes factores de riesgo para enfermedades muy prevalentes, como la cardiopatía isquémica, los accidentes cerebrovasculares, las vasculopatías periféricas ateroscleróticas, entre muchas otras (1). Recientemente, desde hace unos 5 años han surgido nuevos fármacos con una gran efectividad demostrada en la reducción de los niveles de colesterol, especialmente del LDL, se trata de los inhibidores de la proproteína convertasa de subtilisina/kexina tipo 9 (PCSK-9), los cuales evitan que, a nivel hepático, los receptores de LDL sean hidrolizados. Su indicación de uso está definida por la falta de cumplimiento de las metas (específicas según el nivel de riesgo del paciente) de LDL, a pesar del tratamiento con estatinas en la máxima dosis tolerable, junto con ezetimiba, y pueden conseguir una reducción adicional de los niveles de LDL de $60 \%$ aproximadamente. Su concentración máxima en el plasma se alcanza a los 3-4 días y su vida media oscila entre los 11-17 días, por lo que usualmente se utiliza con dosis de cada 2 semanas (2).

\section{Presentación del caso}

Paciente masculino de 67 años con antecedente de diabetes mellitus tipo 2, insulinodependiente y diagnosticado desde hace 15 años, con única complicación microvascular consistente en neuropatía periférica con hipoestesia en dorso de pie y dolor en la región poplítea y sin ninguna complicación macrovascular. Además de esto, presenta pérdida de $15 \mathrm{~kg}$ de peso al inicio de la enfermedad, atribuible a cambios en el estilo de vida (dieta y ejercicio). Al examen físico talla de 1,81 m, peso $101 \mathrm{~kg}$, índice de masa corporal (IMC) de 30,83 kg/m², tensión arterial de 150/88 mm Hg y prueba de monofilamento alterado en dorso de ambos pies. Como abordaje inicial se comienza tratamiento con insulina glargina, metformina 1000 $\mathrm{mg}$, ramipril $5 \mathrm{mg}$, pravastatina, lansoprazol y pregabalina; con valores a los 3 meses de colesterol total $243 \mathrm{mg} / \mathrm{dL}$, colesterol HDL-C $33 \mathrm{mg} / \mathrm{dL}$, triglicéridos $492 \mathrm{mg} / \mathrm{dl}$, LDL $111 \mathrm{mg} /$ dL y HbA1c 7,9 \%.

El paciente suspende la estatina por mialgia, que afecta su calidad de vida. Nuevamente se intentó el uso de atorvastatina $40 \mathrm{mg}$ con intolerancia. Por el perfil del paciente está indicada una estatina de alta potencia con reducción de LDL de más del 
$50 \%$, el cual no se obtuvo por intolerancia a estatina, por lo cual se indica evolocumab $140 \mathrm{mg} / 2$ semanas. Luego de 15 días de tratamiento, el paciente presenta una mejoría en su perfil lipídico, con colesterol total $140 \mathrm{mg} / \mathrm{dL}$, HDL-C $36 \mathrm{mg} /$ dL, LDL-C $45 \mathrm{mg} / \mathrm{dL}$ y triglicéridos $348 \mathrm{mg} / \mathrm{dL}$ y se continúa el tratamiento con evolocumab más fenofibrato por hipertrigliceridemia y ajuste en los medicamentos antidiabéticos.

Luego de 2 meses de tratamiento con evolocumab, el paciente presenta un perfil lipídico de colesterol total $97 \mathrm{mg} / \mathrm{dL}$, HDL-C $34 \mathrm{mg} / \mathrm{dL}$, triglicéridos $194 \mathrm{mg} / \mathrm{dL}$, LDL 24mg/dl. Se decide continuar la dosis de evolocumab a $140 \mathrm{mg} / 2$ semanas. 4 semanas después, el paciente acude a la consulta indicando una dilatación del tiempo de aplicación del medicamento por motivos económicos en intervalos de 1 vez cada 4 semanas, y aporta un perfil lipídico tomado el día anterior a la consulta que arroja como resultado C-LDL de $54 \mathrm{mg} / \mathrm{dL}$. Después de 4 semanas, se toma un nuevo perfil lipídico que reporta colesterol total de $149 \mathrm{mg} / \mathrm{dL}$, HDL-C de $36 \mathrm{mg} / \mathrm{dL}$, LDL-C $79 \mathrm{mg} / \mathrm{dL}$ y triglicéridos de $248 \mathrm{mg} / \mathrm{dL}$.

\section{Discusión}

Según las guías del Journal of the American Medical Association (JAMA), el manejo farmacológico para la disminución del colesterol de baja densidad (LDL-C) está indicado en pacientes con clínica o antecedente de infarto agudo al miocardio, accidente cerebrovascular y muerte cardiovascular, con metas de LDL menores de $70 \mathrm{mg} / \mathrm{dL}$ (o <55 mg/dL según AACE) (1).

El actual caso clínico presenta un paciente clasificado como de alto riesgo cardiovascular, con un $44 \%$ de posibilida- des de presentar un evento cardiovascular mayor en 10 años, esto debido a sus patologías: hipertensión, diabetes y dislipidemia de base (3). Las últimas guías de JAMA para el manejo del colesterol indican que en pacientes con alto riesgo de eventos cardiovasculares mayores está indicado el uso de estatinas de alta intensidad (atorvastatina de $40-80 \mathrm{mg}$ y rosuvastatina de 20-40 mg) con el fin de llevar los niveles de colesterol LDL por debajo de $100 \mathrm{mg} / \mathrm{dL}$ (1); inclusive por debajo de $70 \mathrm{mg} /$ dL por tener más de 10 años de evolución de diabetes, tasa de filtración glomerular (TFG) entre 30 y 60, e hipertensión arterial. Dado que el paciente presentó una marcada intolerancia a la terapia con estatinas y debido a los altos niveles de colesterol, se consideró que la mejor opción terapéutica para el paciente era evolocumab de $140 \mathrm{mg}$ cada 2 semanas, aunque la vida media biológica del evolocumab es en promedio de 11 a 17 días, lo cual justifica los intervalos de aplicación designados en las diferentes guías.

El resultado clínico de este caso sugiere una acción farmacológica tangible con intervalos de dosis más espaciados, ya que con intervalos de inclusive 28 días, el paciente se encuentra en metas adecuadas para la reducción del riesgo cardiovascular, lo cual corrobora la hipótesis de poder utilizar en este caso inhibidores de la PCSK9 (evolocumab) bajo la guía de las metas de LDL en el caso de pacientes con intolerancia a las estatinas (2); además, se presenta como un hallazgo importante para la elaboración de nuevos estudios dirigidos a la aplicación de dosis más espaciadas, más costo-efectivas y, por ende, con menor tasa de efectos adversos asociados a la dosis del fármaco.

\section{Referencias}

1. Alenghat FJ, Davis AM. Management of blood cholesterol. JAMA. 2019;321(8):800-1.

2. Cicero AF, Bove M, Borghi C. Pharmacokinetics, pharmacodynamics and clinical efficacy of non-statin treatments for hypercholesterolemia. Expert Opin Drug Metab Toxicol. 2018;14(1):9-15.
3. American Diabetes Association. Cardiovascular disease and risk management: standards of medical care in diabetes. Diabetes Care. 2019;2(1):S103-23. 